\title{
Descripción Anatómica del Esqueleto de (Isurus oxyrinchus)
}

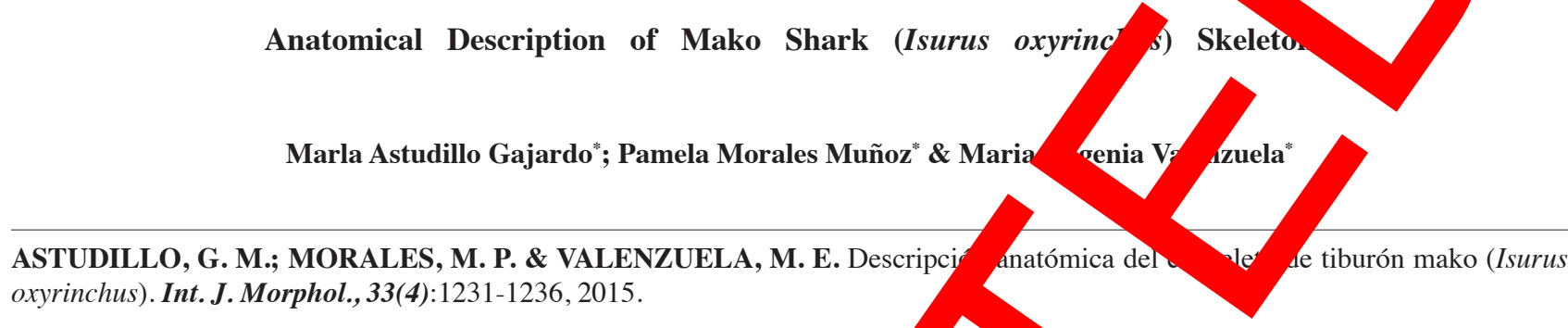 \\ a \\ Sketetor \\ oxyrinchus). Int. J. Morphol., 33(4):1231-1236, 2015. \\ RESUMEN: La especie Isurus oxyrinchus, conocido también com Aburón Mu Marrajo Común, pertenece a la familia \\ Lamnidae, de la clase Condrictria, cuya principal característica es su esqueleto, el cual está co rido completamente por cartílago. Se \\ considera una especie vulnerable según la UICN (Unión Internacional paro- Gonservación de là curaleza) como consecuencia de su \\ utilización en la industria alimentaria y, en el último tiempo, a la ca ceportiva, debido a su gran velocidad. Tiene una distribución \\ cosmopolita, encontrándose en los océanos Pacífico, Atlántico, Índi , así como tambión en el Mar Mediterráneo y Mar Rojo. En Chile \\ se tiene registro de su captura desde el norte hasta la región centro $r$ del país. Son ejo lares de gran tamaño, llegando a medir hasta \\ 3,5 metros y pesar alrededor de $570 \mathrm{~kg}$. El presente trabajo correspo e a un estudio anat iico de un ejemplar juvenil de Tiburón Mako, \\ obtenido por pesca incidental en las costas del Maule, Chile. La des ción del esquele se realizó de manera sistemática por segmen- \\ tos: cráneo, columna y aletas. Se obtuvo un registro fotográfico de los ados. De a rdo lo observado, podemos indicar que existe \\ gran similitud con lo descrito en el Tiburón Espinoso y Tiburón Blanco, nariaciones de forma en el esqueleto de esta \\ especie, que corresponderían a las características que fieren su aptitud para nadar a gran velocidad. La presente descripción, \\ permite ampliar el conocimiento de la anatomía del Tib nn Mran información de esta especie poco estudiada. \\ PALABRAS CLAVE: Tiburón Mako; Esqueleto, on ctios.
}

\section{INTRODUCCIÓN}

La especie Isurus oxyrinchion or do también como Tiburón Mako o Mar $\delta$, perte a la familia Lamnidae, del orden Lamp rmes, de la clà Sondrictia (Guisande, 2013). A la mi 'omilia pertenece tomíbién el Tiburón Blanco (Del N al \& z, 2013). Se considera una especie vulnerablêsegún la Un Unión Internacional para la Consery ión de la Naturalo Cailliet et al., 2009), ya que se mercializa como alimento, y en el último tiempo se b nstaurad caza deportiva de este ejemplar, esto deb a que onsideramn animal muy rápido, alcanzando $\mathrm{ka}$ dad de $124 \mathrm{kr}$, en donde la estructura anatómica de lá a caudal a forma fusiforme de su cuerpo un rol in tar La aleta caudal posee un pedú alo ca al compris $\sigma$ dorso-ventralmente, facilita $\delta$ así el vimiento lateral de la aleta, reduciendo la stencia gham-Soliar, 2005). Las caracteris gr permiten reconocer al Tiburón Mako son: cuerpo fuss e, hocico cónico y puntiagudo, boca grande y, la principal ca rerística de esta especie es que sus dientes se encuentran per, nentemente a la vista. Posee 5 hendiduras branquiales, aletas escapulares medianas, de extremo ligeramente redondeado, aleta dorsal que se origina justo caudal a las aletas escapulares y finalmente la aleta caudal posee grandes lóbulos, siendo el dorsal ligeramente mayor que el ventral (Lamilla \& Bustamante, 2005).

Tiene una distribución bastante amplia, costera y oceánica, alrededor de todo el mundo, entre las latitudes $50^{\circ} \mathrm{N}$ a $50^{\circ} \mathrm{S}$ (Cailliet et al.), en aguas templadas y tropicales, por lo que en Chile se podría encontrar desde la zona norte a centro-sur del país, excluyendo el extremo sur (Valeiras \& Abad, 2006). Es una especie que en su mayoría es capturado incidentalmente en la pesca de pez espada y atún (Ribot, 2003). Lamilla \& Bustamante indican que usualmente se extrae solamente las aletas, que es lo que tiene mayor valor comercial, y el resto normalmente se descarta debido al bajo precio de la carne, por lo anterior, no existe un registro de su captura (Bustamante \& Lamilla, 2006). Es importante destacar que este animal constituye un riesgo para las personas tanto en pesca incidental como deportiva ya 
que se conocen registros informales de ataques a seres humanos. Por otra parte, existen escasos estudios que describan esta especie, solo algunos sobre migraciones y comportamiento (Medellín-Ortiz et al., 2008).

El objetivo fue conocer la anatomía del esqueleto cartilaginoso de esta especie, entregando información valiosa para su reconocimiento como también para generar una base para futuros trabajos sobre esta especie u otras especies de tiburones.

\section{MATERIAL Y MÉTODO}

Se utilizó un cadáver de Tiburón Mako, macho, juvenil, de 1,70 m de largo y $21 \mathrm{~kg}$ de peso (eviscerado), obtenido por pesca incidental en las costas de la localidad de Duao, Región del Maule, Chile, el cual fue donado a la Unidad de Anatomía Veterinaria de la Universidad Santo Tomás, sede Talca. Mediante la utilización de instrumental de disección de rutina se extrajo el tegumento y gran parte de tejido muscular, para luego conservar la muestra en form - lina al $1 \%$, y así continuar con el trabajo de limpieza del e tue
leto. La disección se efectuó de craneal a caudal, realiza un estudio descriptivo de las principales estructur cartilaginosas que conforman el esqueleto: cráneo branquias, hioides, columna, aletas pectorales, aleta pél a caudal. Se realizó registro fotográfico de cad

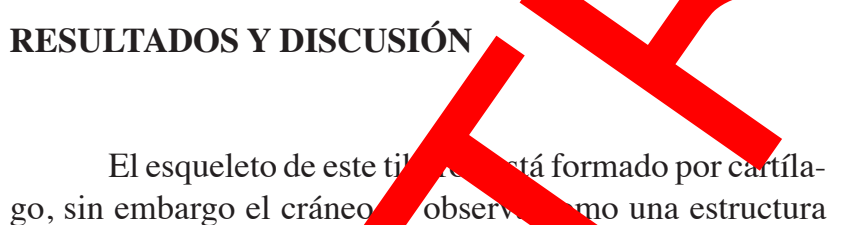
de mayor densidad tisular que el resto vuerpo, lo que hace suponer que dicl zona presenta may zantidad de impregnación de cal, tal como se describe en otras especies de condrictio ardong 12).

Cráneo. Se divio segmen ondrocráneo y Esplacnocráneo. El Co. cráneo cl que protege el encéfalo y órg sentia se encuentra el rostrum, que se syecta tralmente, cual contiene la cavidad prece oral, sim en Tiburón Espinoso, otre necie de muncmo muente de observar en las costas de r. aís (Quintanilla, 2007). En el caso del Tiburón Mako trum posee dos bandas tubulares de cartílago dorsal que nen rostralmente, y una tercera banda tubular, medial y vontral a las anteriores, que correspondería a la carina rostral, que también se une a ellas en el extremo rostral, por lo cual el rostrum se observa abierto lateral- mente, lo que difiere con lo descrito ep en donde el rostrum se sostiene en a carina vè y se observa cerrado lateral y ventr nente con dos Iras rostrales pequeñas (De Iullis \& Pu 2007). El par d ápsulas nasales se encuentra lateral en se del ros $\mathrm{m}, \mathrm{y}$ los nares de cada cápsy se encuen or atados ventralmente (Fig. 1). P erior a los cápsui asales se encuentra la región ópł , en don la órbita no es cerrada por completo, dorsaln aposee oceso ant jital (rostral), cresta supraorbital(dors oceso po rbital (caudal) (Fig. 2). Medial el proceso orbital encuentra el foramen para el sio oftálmico s. ral, el cual emerge por dicho for ara luego ramificarse dentro del rostrum, lo que dify con blicado en el Tiburón Espinoso, en donde el nervio mena do emerge por una serie de forámenoc nequeños ubicad n la cresta supraorbital (De Iull rurerà). El foramen óptico se encuentra en la pared $\mathrm{m}$ al de la órbit El Esplacnocráneo incluye 7 arcos cerales: arco ndibular, arco hioideo y 5 arcos b quiales. Los arc mandibular e hioideo están altamente dificados par a uso en la captura de presas, las cuales co. palmente en peces óseos y constituyen alrededor ae un $87 \%$ de la dieta del Tiburón Mako (López et (nona). Los arcos branquiales le dan soporte a los septos in oram des, además de la lengua. El arco mandibular el más grande; el cartílago hiomandibular es el único elehento cartilaginoso que lo relaciona con el cráneo, ya que stralmente se encuentra unido sólo por musculatura y 1 ias, este tipo de articulación se denomina hiostílica Teisaire et al., 2010). Los dos cartílagos Palatocuadrados se fusionan rostralmente para formar la mandíbula superior. La otra mitad del arco mandibular forma la mandíbula inferior, la cual está compuesta por un par de cartílagos mandibulares fusionados rostralmente (Fig. 2). El arco mandibular es el que le da soporte a los dientes, las cuales están en constante producción, llegando a formarse 20.000 dientes en los primeros 25 años del ejemplar (MendozaVargas, 2009). En cada hemi mandíbula, se observan dos filas dentales, sin embargo, al extraer la mucosa de la región se observan otras cinco filas dentales al interior de la mandíbula, siendo las más superficiales de consistencia firme, y los dientes que se encuentran en profundidad bastante más frágiles y flexibles (Fig. 3). Los dientes son una característica que permite identificar a una especie detiburón, en el caso del Tiburón Mako, los dientes son de base estrecha con bordes laterales lisos (Lamilla, 2005), a diferencia del Tiburón Blanco, cuya base es ancha y los bordes laterales son aserrados (Klimley \& Anley, 1996). El arco hioideo está modificado para sostener el arco mandibular, el segmento dorsal de este arco corresponde a los cartílagos hiomandibular (uno a cada lado) los cuales se encuentran relacionados con el cráneo. El cartílago hiomandibular articula con el cartílago queratohiodes, el cual, a su vez, articu- 


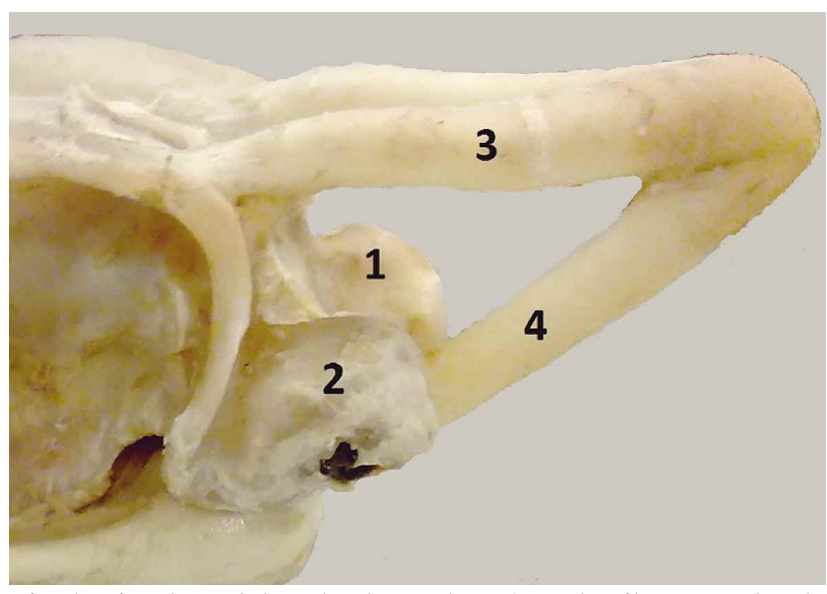

Fig. 1. Vista lateral derecha de condrocráneo de Tiburón Mako. 1Cápsula nasal izquierda. 2- Cápsula nasal derecha. 3- Rostrum. 4Carina rostral.

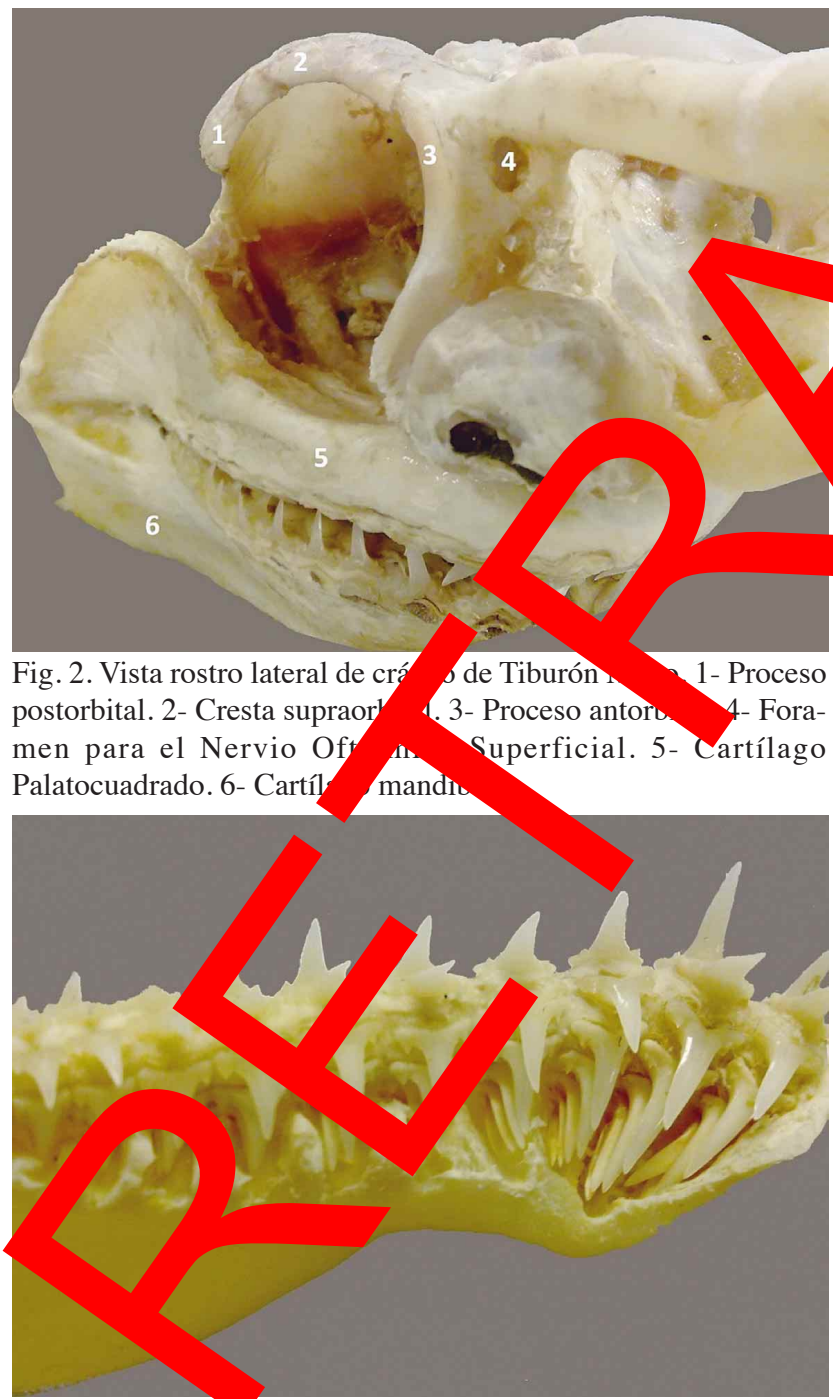

Fig. 3. Vista medıà de cartílago mandibular izquierdo (hemi mandíbula inferior izquierda) de Tiburón Mako. Se observa filas dentales y formación de nuevos dientes al interior de la mandíbula. la con el cartílago basihioides (Fig imo presenta una leve diferencia con lo descr en el Tibu Espinoso (De Iullis \& Pulerà), en el que basihiodes es m alargado, en cambio en el Tiburón $\mathrm{n}$. es más corto y 1 ondeado. Los arcos restantes son los brà. iales, los cy están formados, de dorsal ventral, po rtíl os pares: faringobraquial, e ranquisl y quo branquial, Ventralmente los ar se comp an por 3 pares de cartílagos hipobranquiales, cartíla basibrar ciales que se en-
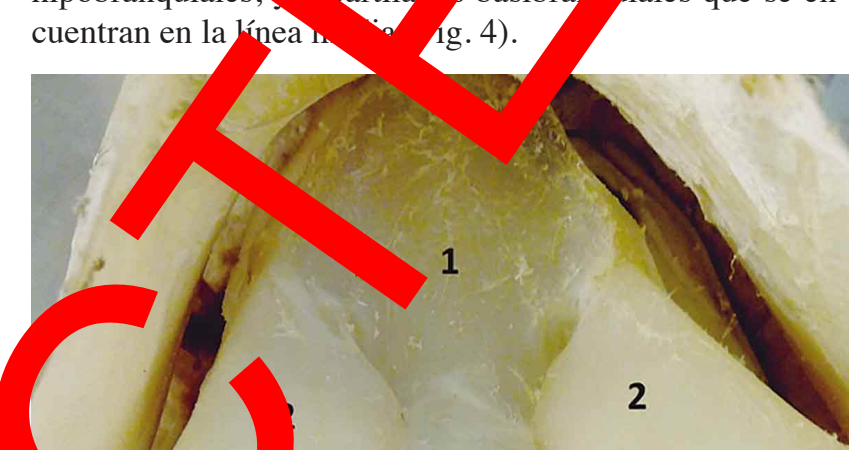

3

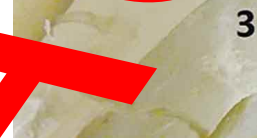

4

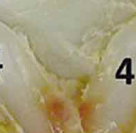

3
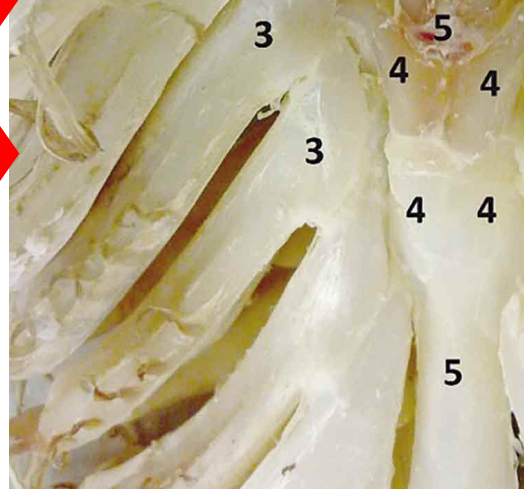

3

4

3

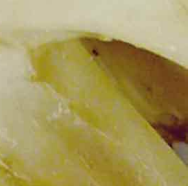

3
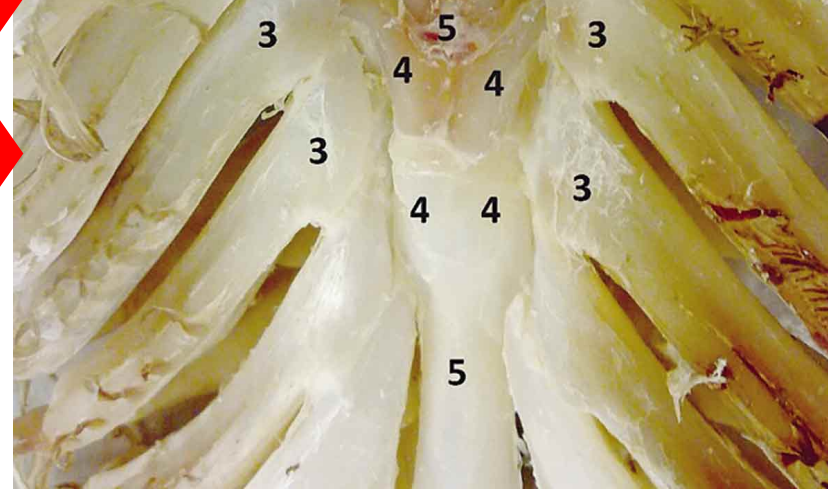

Fig. 4. Vista ventral de arco hioideo y arcos branquiales de Tiburón Mako. 1- Basihioides. 2- Queratohioides. 3- Queratobranquial. 4- Hipobranquial. 5- Basibranquial.

Columna. Hay 2 tipos de vértebras en la columna: las del tronco y las caudales. Cada vértebra posee un cuerpo vertebral, o centrum (ventral), los cuales al unirse forman un canal en cuyo interior se encuentra protegida la notocorda. Dorsal a cada cuerpo vertebral se observa una proyección denominada plato neural triangular, cuya base está orientada hacia el cuerpo vertebral, entre platos neurales consecutivos se encuentran placas intercalares, también triangulares, pero invertidas en relación a los platos neurales, así forman el arco neural, canal en cuyo interior se encuentra protegida la médula espinal. Las vértebras del tronco, a diferencia de las caudales, poseen una proyección, ventral y la- 
teral al cuerpo vertebral, llamada Basapófisis (Fig. 5), en cambio, las vértebras caudales, poseen ventral al cuerpo un arco hemal para el paso de la arteria y vena caudal, lo que concuerda con lo descrito en el Tiburón Espinoso (De Iullis \& Pulerà).

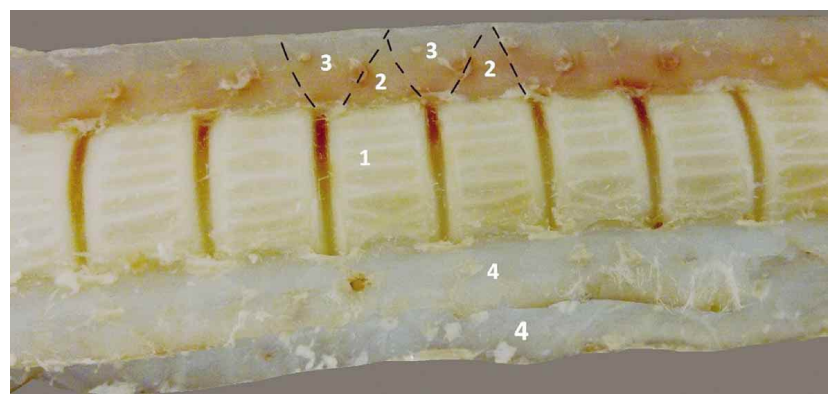

Fig. 5. Vista lateral derecha de vértebras del tronco de Tiburón Mako. 1- Cuerpo vertebral. 2- Plato neural. 3- Plato intercalar. 4Basapofisis derecha e izquierda. Las líneas punteadas indican la forma triangular que poseen estos delgados cartílagos.

Aletas. Las aletas pectorales están unidas entre sí por un grueso cartílago en forma de "U". La porción entre ellas corresponde a la Barra Coracoide. La parte que se ex dorsalmente desde la unión entre las aletas correspon al proceso escapular (Fig. 6). En la aleta se reconocen tres tructuras denominadas pterigioforas basales que articula, con el proceso escapular mediante la superfi anoidea: Propterigio, Mesopterigio y Metapterigio g.7). t tal en la aleta se encuentran las pterigioforas diales, que corresponden a pequeñar rras de cartílago, paralelas entre sí, dispue. tas en 2 filas. Finalmente, en 18 orción distal de la aleta se encu ran las queratotriquias, que correspor na fila de barras de cartílago alar entre sí (Fig. 8). Esta distribución es sinh lar a los descrito en é ciburón Espinoso (De Iullis \& Pulerà)

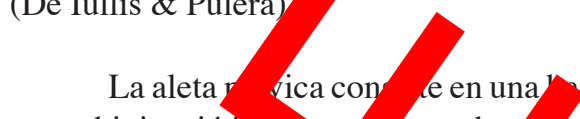
rra pubioisquiátio. en cada of emo posee un proceso 0 . A dif ncia de la ale oral, po dos pterigi oras b ales: Prop rigio y Meta rigio, ad ás de nterigioforas radia Existe Hencrum las aletas pélvica. cho y hembra, en el macho el Metapte de cada lado, presenta una proyección cau ra formar los Fórceps, que corresponde ar gano copulador (Fig. 9), esto basados en el esquema publicado por De Iullis \& Pulerà.
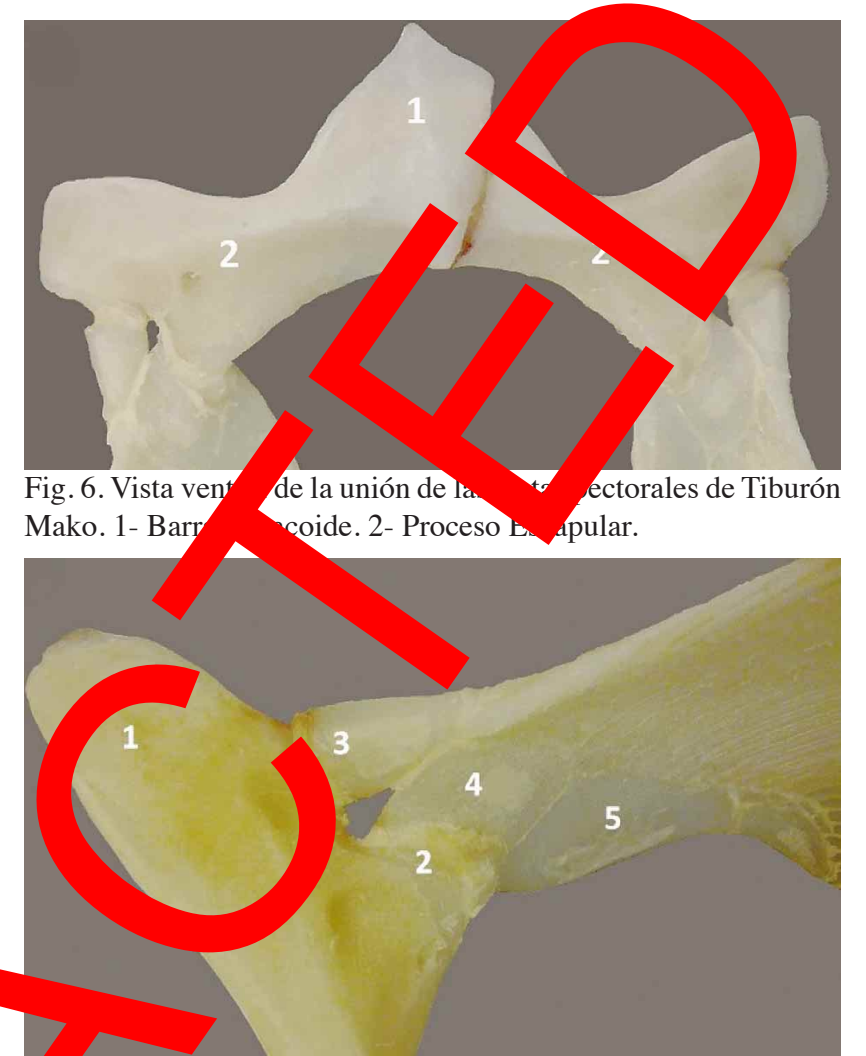

7. Vista ventral de la articulación de la aleta pectoral y terigioforas basales de Tiburón Mako. 1- Proceso Escapular. 2uperficie Glenoídea. 3- Propterigio. 4- Mesopterigio. 5apterigio.

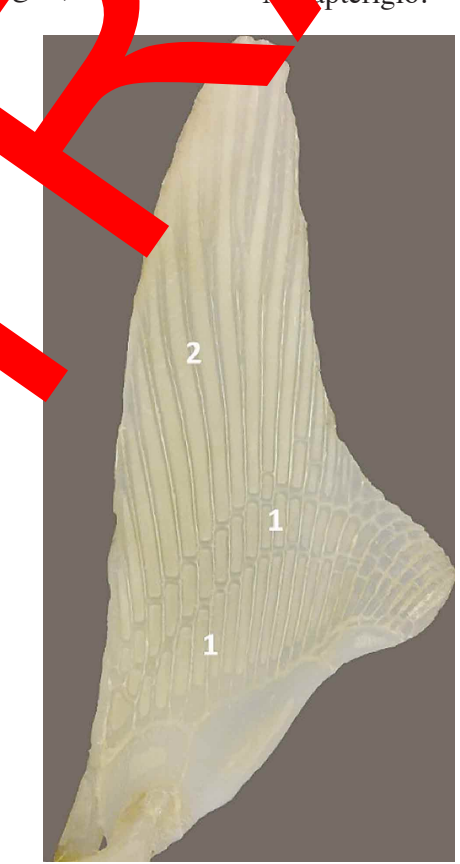

Fig. 8. Vista medial de aleta pectoral izquierda de Tiburón Mako. 1- Pterigioforas radiales. 2Queratotriquias.

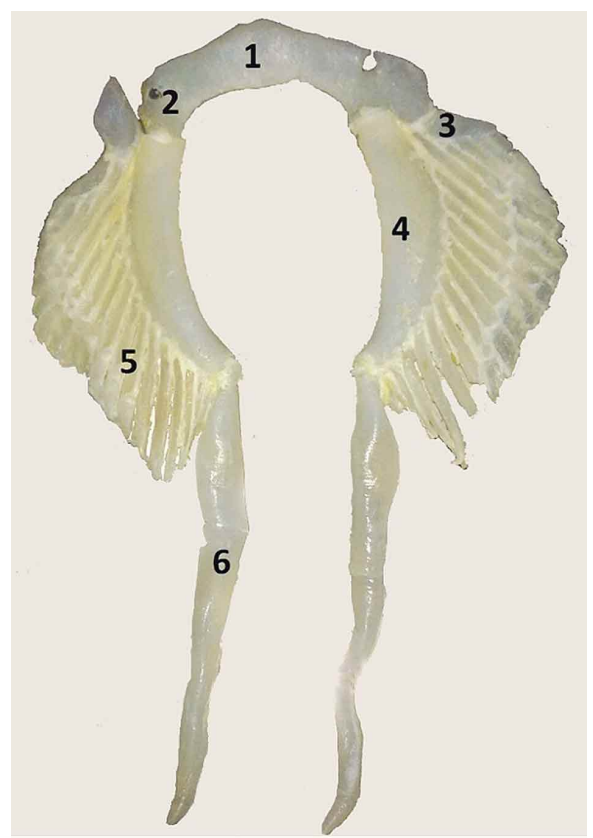

Fig. 9. Vista ventral de aleta pélvica de macho de Tiburón Mako. 1- Barra Isquiopubiana. 2Proceso Iliaco. 3- Propterigio. 4- Metapterigio. 5- Pterigioforas radiales. 6- Fórceps. 
Finalmente, la aleta caudal está conformada por los cuerpos vertebrales, arcos hemales y pterigioforas radiales (Fig. 10). Es importante mencionar que en el espécimen completo la aleta caudal se observa conformada por dos lobos: dorsal y ventral, sin embargo solo el lobo dorsal está compuesto por tejido cartilaginoso y, por tanto, formando parte del esqueleto, el lobo ventral posee solo tejido fibroso y muscular. Debido a lo anterior, los cuerpos vertebrales se observan incluidos dentro del lobo dorsal cartilaginoso, llegando hasta el final aguzado de la aleta, esta descripción concuerda con lo observado en el Tiburón Blanco (LinghamSoliar).

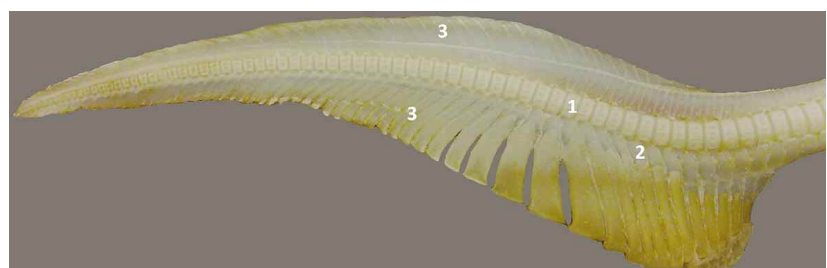

Fig. 10. Vista lateral derecha de aleta caudal de Tiburón Mako. 1Cuerpo vertebral. 2- Arco Hemal. 3- Pterigioforas radiales.

Considered to be a vulnerable speci to the IUCN (International Union for Conservati of Nature), a esult of its extensive use in food industry an anting sports duc its high speed. It has a cosmopolitan distr ion, being found in Pacific, Atlantic and Indian Oceans, the M ranean Sea a the Red Sea. In Chile, a variety of $t^{\prime}$ species distribut from the North to the Central-Soy egions of the cording to industrial fishing data) ecimens large in reaching 3.5 $\mathrm{m}$ and around $570 \mathrm{~kg}$ veigh. Th $\mathrm{m}$ of this work was to perform a gross anatomy stu a juve Mako Sh obtained by incidental fishing off the co f ale, Chile ne description of the skeleton was ced out in atematic ay begments: skull, spine and fin photographic rea specimen was obtained. According results, there is gimilarity with what was describe or th ckly shark and white shark, except for some shape riations in skeleton. These differences could be explained by its abilins swim at high speed. The present decallows expandin knowledge of the anatomy of the ко Shark, generating more information on this species.

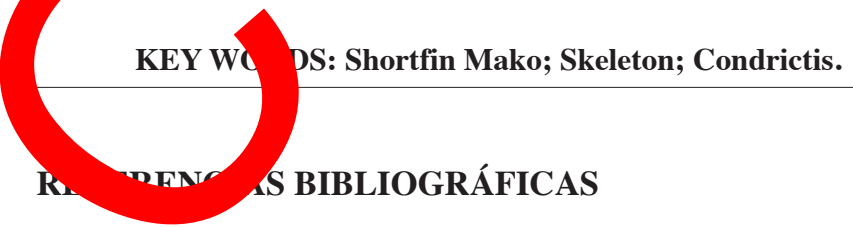

\section{CONCLUSIONES}

De acuerdo a la disección y estudio realizado posible describir en detalle el esqueleto Tiburón Mako (Isurus oxyrinchus). Se oncluy ue según lo observado en este ejemplar exist gran simj ad con-lo descrito en el Tiburón Espinoso burón Blanco (Cacharodon carcharı. po olemente con otras especies de condrictios. $\mathrm{Cembarg}$ isten algunas diferencias a nivel del cráne omo: rostrum a to lateralmente, foramen para el n oftálmico superfio il se encuentra medial en el pr so an ital, dientes de base estrecha y bordes lisos y vasihioides in orto y redondeado.

Este estud descriptivo permite ampliar el conocimiento anatóm referen l esqueleto de los condrictios, especialment el Tibur Mako, esnecie presente en nuestro país y quo vido casament studiada. Además esta información podín utilizada mo base para la realización de sestudio ar smía comparada.

IUDILL RALES, M. P. \& VALENZUELA, 1. Anat ical description of Mako Shark (Isurus oxyrinchus) skele ..J.Morphol., 33(4):1231-1236, 2015.

SUMn Y: The species Isurus oxyrinchus, also known as Mako Shark or commonly as Shortfin Mako, belongs to the Lamnidae family, of the Condrictis class. The main anatomical feature of this class is a skeleton fully composed of cartilage.

\section{-}

, C. \& Lamilla, J. Estado actual de la pesquería y biología de los condricthyes en Chile. II Taller de Cooperación Internacional - Tiburones del Pacífico: ¿Manejo local o conjunto? Valdivia, Universidad Austral de Chile, 2006. pp.2666.

Cailliet, G.; Cavanagh, R.; Kulka, D.; Stevens, J.; Soldo, A.; Clo, S.; Macias, D.; Baum, J.; Kohin, S.; Duarte, A.; Holtzhausen, J.; Acuña, E.; Amorim, A. \& Domingo, A. Isurus oxyrinchus. The IUCN Red List of Threatened Species. Version 2014.2. 2009. Disponible en: www.iucnredlist.org

De Iullis, G. \& Pulerà, D. The Dissection of Vertebrates: a Laboratory Manual. Londres, Academic Press, 2007. pp.2735 .

Del Moral, L. \& Pérez, G. Tiburones, Rayas y Quimeras de México. CONABIO. Biodiversitas, 111:1-6, 2013.

Guisande, C.; Pascual, P.; Baro, J.; Granado, C.; Acuña, A.; Manjarrés, A. \& Pelayo, P. Tiburones, rayas, quimeras, lampreas y mixínidos de la costa atlántica de la Península Ibérica y Canaria. Madrid, Ediciones Diaz de Santos, 2013. p.22

Kardong, K. The vertebrates story. In: Vertebrates: comparative anatomy, function, evolution. ( $6^{\circ}$ ed.). Nueva York, McGrawHill, 2012.

Kimley, A. \& Anley, D. Great White Sharks: The Biology of Carcharodon carcharias. San Diego, Academic Press, 1996. pp.9-19. 
Lamilla, J. Bycatch: Tiburones en peligro. Oceana, 11:1-14, 2005.

Lamilla, J. \& Bustamante, C. Guía para el reconocimiento de: tiburones, rayas y quimeras de Chile. Oceana, 17:28-9, 2005.

Lingham-Soliar, T. Caudal Fin in the White Shark, Carcharodon carcharias (lamnidae): a dynamic propeller for fast, efficient swimming. J. Morph., 264(2):233-52, 2005.

López, S.; Meléndez, R. \& Barría, P. Alimentación del tiburón marrajo Isurus oxyrinchus Rafinesque, 1810 (Limniformes: Lamnidae) en el pacífico suroriental.Rev. Biol.Mar. Oceanogr., 44(2):439-451, 2009.

Medellín-Ortiz, A.; Kohin, S.; Dewar, O. \& Block, B. Movimientos del tiburón mako (Isurus oxyrinchus) en el Pacífico Noreste. México DF, III Simposium Nacional de Tiburones y Rayas. Facultad de Ciencias, Universidad Nacional Autónoma de México, 2009. pp.142-26.

Mendoza-Vargas, O. Dentición en Tiburones. III Simposium Nacional de Tiburones y Rayas. México D.F., Facultad de Ciencias, Universidad Nacional Autónoma de México, 2009.

Quintanilla, J. Descripción y determinación de estados de madurez gonadal y gestación en el Tollos de Cachos medi utilización de la técnica ecográfica y complementarias moria para optar al título de Médico Veterinario. Valdi Facultad de Ciencias Veterinarias, Universidad Austral Chile, 2007.

Ribot, M. Edad y Crecimiento del Tiburón Mo Costa Occidental de Baja California S obtener el grado de Maestro en Ciene con espe recursos marinos. La Paz, Instituto Polit oN onal, Centro interdisciplinario de ciencias inas,

Teisaire, E.; Nieto, O.; Roldán, I.; oa, Z.; Aragón, M. cóa, A. Guía de Trabajos Práct s Inatomía Comparada de Vertebrados. Reduca (Bi gía). Ser, J, 3(6):1-15, 2010.

Valeiras, J. \& Abad, E. I ajo dientuso. Field \al. Madrid, International Com sion For The Conservation Of Atlantic Tunas, 2010.

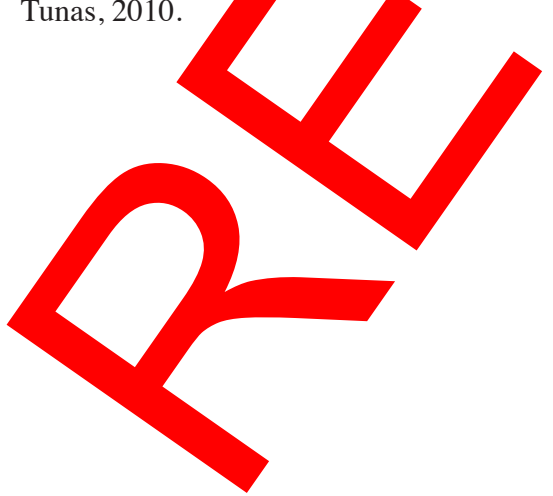

Dirección para Correspondencia: Marla Astudillo Gajardo Escuela de Medicina Veterinaria Facultad de Medicina Veterinariz Universidad Santo Tomás

Talca

CHILE

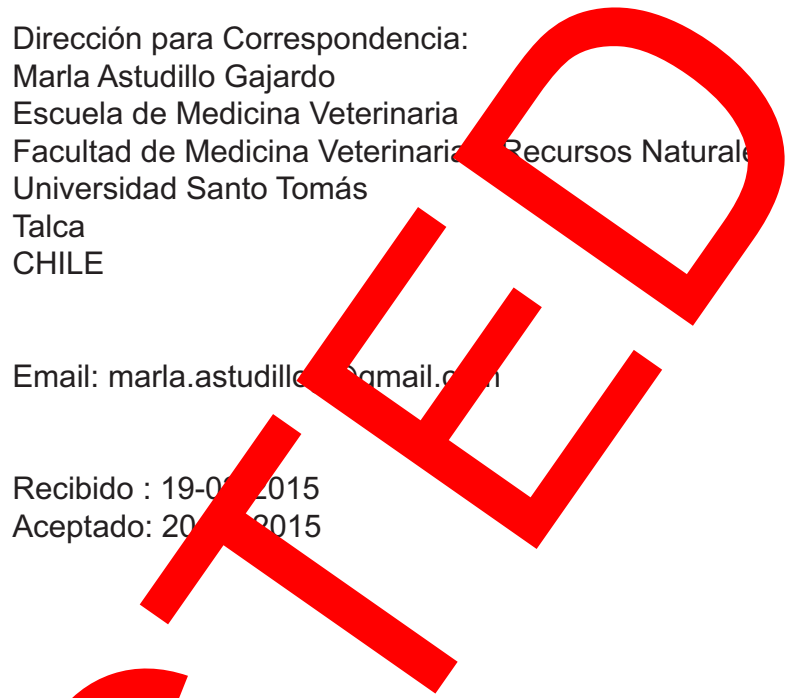

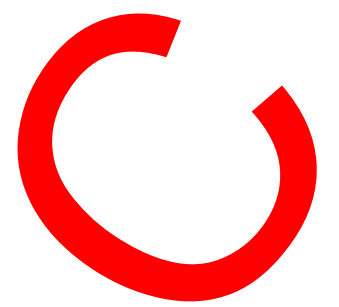

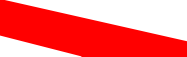

